\title{
Latest Results from PHOBOS
}

\author{
D J Hofman ${ }^{6}$ for the PHOBOS Collaboration
}

\author{
B.Alver ${ }^{4}$, B.B.Back ${ }^{1}$, M.D.Baker ${ }^{2}$, M.Ballintijn ${ }^{4}$, D.S.Barton ${ }^{2}$, \\ R.R.Betts ${ }^{6}$, A.A.Bickley ${ }^{7}$, R.Bindel ${ }^{7}$, W.Busza ${ }^{4}$, A.Carroll ${ }^{2}$, \\ Z.Chai $^{2}$, V.Chetluru ${ }^{6}$, M.P.Decowski ${ }^{4}$, E.García ${ }^{6}$, N.George ${ }^{2}$, \\ T.Gburek $^{3}$, K.Gulbrandsen ${ }^{4}$, C.Halliwell ${ }^{6}$, J.Hamblen ${ }^{8}$, \\ I.Harnarine $^{6}$, M.Hauer ${ }^{2}$, C.Henderson ${ }^{4}$, D.J.Hofman ${ }^{6}$, \\ R.S.Hollis ${ }^{6}$, R.Hołyński ${ }^{3}$, B.Holzman ${ }^{2}$, A.Iordanova ${ }^{6}$, \\ E.Johnson ${ }^{8}$, J.L.Kane ${ }^{4}$, N.Khan ${ }^{8}$, P.Kulinich ${ }^{4}$, C.M.Kuo ${ }^{5}$, \\ W.Li ${ }^{4}$, W.T.Lin ${ }^{5}$, C.Loizides ${ }^{4}$, S.Manly ${ }^{8}$, A.C.Mignerey ${ }^{7}$, \\ R.Nouicer ${ }^{2}$, A.Olszewski ${ }^{3}$, R.Pak ${ }^{2}$, C.Reed ${ }^{4}$, E.Richardson ${ }^{7}$, \\ C.Roland $^{4}$, G.Roland ${ }^{4}$, J.Sagerer ${ }^{6}$, H.Seals ${ }^{2}$, I.Sedykh ${ }^{2}$, \\ C.E.Smith ${ }^{6}$, M.A.Stankiewicz ${ }^{2}$, P.Steinberg ${ }^{2}$, G.S.F.Stephans ${ }^{4}$, \\ A.Sukhanov ${ }^{2}$, A.Szostak ${ }^{2}$, M.B.Tonjes ${ }^{7}$, A.Trzupek ${ }^{3}$, C.Vale ${ }^{4}$, \\ G.J.van Nieuwenhuizen ${ }^{4}$, S.S.Vaurynovich ${ }^{4}$, R.Verdier ${ }^{4}$, \\ G.I.Veres ${ }^{4}$, P.Walters ${ }^{8}$, E.Wenger ${ }^{4}$, D.Willhelm ${ }^{7}$, F.L.H.Wolfs ${ }^{8}$, \\ B.Wosiek ${ }^{3}$, K.Woźniak ${ }^{3}$, S.Wyngaardt ${ }^{2}$, B.Wysłouch ${ }^{4}$ \\ ${ }^{1}$ Argonne National Laboratory, Argonne, IL 60439-4843, USA \\ 2 Brookhaven National Laboratory, Upton, NY 11973-5000, USA \\ ${ }^{3}$ Institute of Nuclear Physics PAN, Kraków, Poland \\ ${ }^{4}$ Massachusetts Institute of Technology, Cambridge, MA 02139-4307, USA \\ ${ }^{5}$ National Central University, Chung-Li, Taiwan \\ ${ }^{6}$ University of Illinois at Chicago, Chicago, IL 60607-7059, USA \\ ${ }^{7}$ University of Maryland, College Park, MD 20742, USA \\ ${ }^{8}$ University of Rochester, Rochester, NY 14627, USA \\ E-mail: hofman@uic.edu
}

\begin{abstract}
This manuscript contains a summary of the latest physics results from PHOBOS, as reported at Quark Matter 2006. Highlights include the first measurement from PHOBOS of dynamical elliptic flow fluctuations as well as an explanation of their possible origin, two-particle correlations, identified particle ratios, identified particle spectra and the latest results in global charged particle production.
\end{abstract}

PACS numbers: 25.75.-q

Submitted to: J. Phys. G: Nucl. Part. Phys. 
Over the last year, PHOBOS [1] has continued to analyze the large dataset obtained from the first five runs (2000-2005) of the Relativistic Heavy Ion Collider (RHIC) at Brookhaven National Laboratory. To a large extent, one of the primary goals of PHOBOS to obtain a broad survey of the global properites of charged particle production is nearing completion. We report two new results in this area, the first for the lowest energy $\mathrm{Cu}+\mathrm{Cu}$ collisions, $\sqrt{s_{\mathrm{NN}}}=22.4 \mathrm{GeV}$, and the second for the peripheral yields of midrapidity charged particle emission in $\mathrm{Au}+\mathrm{Au}$. In both cases the new data has followed the striking scaling rules reported in the past by PHOBOS [2, 3, 4, in particular the energy-independence of particle production when viewed in the rest-frame of one of the colliding nuclei [5] and the factorization of the energy and centrality dependence of midrapidity charged particle yields [6, 7]. We also report final results for identified hadron transverse momentum spectra down to very low $p_{T}$ in $\mathrm{Au}+\mathrm{Au}$ collisions at $\sqrt{s_{\mathrm{NN}}}=62.4 \mathrm{GeV}$ as well as new results for antiparticle to particle ratios in $\mathrm{Cu}+\mathrm{Cu}$ collisions [8]. The measurement of both elliptic and directed flow of charged particles over a uniquely large range in pseudorapidity $(-5.4<\eta<5.4)$ has been an area of active analysis in PHOBOS from the very first $\mathrm{Au}+\mathrm{Au}$ collisions [9, 10]. The large elliptic flow signals $\left(v_{2}\right)$ measured near midrapidity at full RHIC energies, which are in agreement with hydrodynamical model calculations of a relativistic hydrodynamic fluid, have provided strong credence for our current view that we are producing a strongly interacting state of matter that reaches equilibration early in the collision process. The comparison between $\mathrm{Cu}+\mathrm{Cu}$ and $\mathrm{Au}+\mathrm{Au}$ of $v_{2}(\eta)$ and mid-rapidity $v_{2}\left(p_{T}\right)$ [11] further strengthens our understanding of the PHOBOS result reported at Quark Matter 2005 [12]: the $v_{2}$ results for both systems can be consistently unified when scaled by the "participant eccentricity", $\left\langle\varepsilon_{\text {part }}\right\rangle$, where $\varepsilon_{\text {part }}$ is defined event-by-event as the initial overlap eccentricity measured in the rotated "participant" frame where that eccentricity is maximized. Furthermore, the importance of the initial event-by-event interaction points of the participant nucleons as defined by $\left\langle\varepsilon_{\text {part }}\right\rangle$, comes into clear focus when the fluctuations of this quantity are calculated and compared to the newly measured $v_{2}$ dynamical fluctuations in $\mathrm{Au}+\mathrm{Au}$ [13, 14]. On the two particle correlations front, we present new data showing correlations between particles in $\mathrm{p}+\mathrm{p}$ and $\mathrm{Cu}+\mathrm{Cu}$ data at $\sqrt{s_{\mathrm{NN}}}=200 \mathrm{GeV}$ over a uniquely large region of $\Delta \eta$ and $\Delta \phi[15]$. We also present the first physics results of a study of the centrality dependence of the shortrange pseudorapidity correlations as measured by the effective cluster size, $K_{\text {eff }}$. Studies of cluster production indicate that the isotropic cluster model produces a cluster width in pseudorapidity that is larger than found in the resonance cascade model. PHOBOS also reported on the latest results of two ongoing studies of multiplicity fluctuations in $\mathrm{Au}+\mathrm{Au}$ collisions, in particular the search for enhanced $d N_{\mathrm{ch}} / d \eta$ fluctuations and the foward-backward multiplicity fluctuations in the context of two models of cluster production [16]. The current upper limit for the fraction of events with large deviations in the $d N_{\mathrm{ch}} / d \eta$ distribution of $200 \mathrm{GeV} \mathrm{Au}+\mathrm{Au}$ collisions is $10^{-5}$. In conclusion, we will give a brief overview of the future direction of the PHOBOS research program.

One of the primary motivations of colliding $\mathrm{Cu}+\mathrm{Cu}$ and $\mathrm{Au}+\mathrm{Au}$ nuclei at $\mathrm{RHIC}$ 


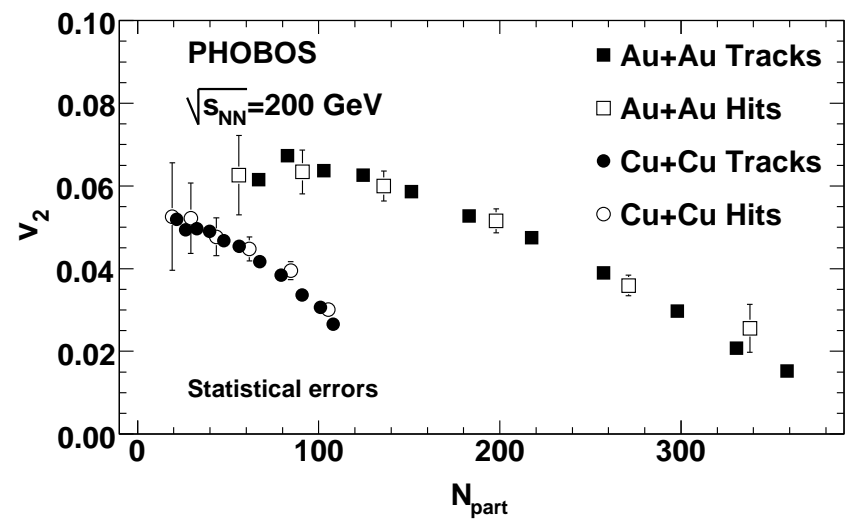

Figure 1. Magnitude of the average elliptic flow coefficient, $v_{2}$, at midrapidity as a function of centrality $\left(N_{\text {part }}\right)$ for $\mathrm{Cu}+\mathrm{Cu}$ and $\mathrm{Au}+\mathrm{Au}$ collisions at $\sqrt{s_{\mathrm{NN}}}=200 \mathrm{GeV}$.

was to enable a detailed study of the effect of system size on all measurable physics observables. The study of elliptic flow, with its sensitivity to the collectivity of the produced matter at very early times of the collision, is a physics measurement of particular interest. The result of this analysis and comparison for $\sqrt{s_{\mathrm{NN}}}=200 \mathrm{GeV}$ is shown in Figure 1 for the PHOBOS hit-based and track-based elliptic flow analysis, as a function of centrality defined by the number of participants, $N_{\text {part }}$. Two important features are immediately evident. First, the magnitude of flow in the smaller $\mathrm{Cu}+\mathrm{Cu}$ system is large and qualitatively follows a similar trend with centrality as seen in the larger $\mathrm{Au}+\mathrm{Au}$ system. Second, even for the most central collisions in $\mathrm{Cu}+\mathrm{Cu}$, the magnitude of $v_{2}$ is substantial, and exceeds that seen in central $\mathrm{Au}+\mathrm{Au}$.

In the most intuitive picture, our understanding of elliptic flow is that the magnitude of the final azimuthal angular distribution of produced particles relative to the reaction plane is a consequence primarily of the initial overlap eccentricity of the colliding nuclei. If this picture is correct, the elliptic flow results for both $\mathrm{Cu}+\mathrm{Cu}$ and $\mathrm{Au}+\mathrm{Au}$ should be compatible if each is scaled by the proper eccentricity. We have proposed that the most relevant eccentricity is not the one calculated relative to the initial impact parameter vector, which we will call the 'standard' calculation $\left\langle\epsilon_{\text {std }}\right\rangle$, but instead the eccentricity calculated event-by-event after rotating into the frame of reference that maximizes the eccentricity defined by the participant nucleon interaction points, $\left\langle\epsilon_{\text {part }}\right\rangle$ [12, 17]. The difference between these calculations is shown in Figure 2, and deviations are clearly evident for smaller number of participants in $\mathrm{Au}+\mathrm{Au}$ and all centralities of $\mathrm{Cu}+\mathrm{Cu}$ collisions, a result that illustrates the importance of finite-number fluctuations of the participant interaction points. This result is robust to the details of the Glauber Monte Carlo simulation, as indicated by the bands which show the $90 \%$ C.L. systematic errors. When the $v_{2}$ data of Figure 1 is scaled by $\left\langle\epsilon_{\text {part }}\right\rangle$, the two very different systems are unified on a single curve as shown in the right-hand side of Figure 2.

The unification of the elliptic flow results in $\mathrm{Cu}+\mathrm{Cu}$ and $\mathrm{Au}+\mathrm{Au}$ collisions when scaled by the participant eccentricity holds not only for the average value of $v_{2}$ at 

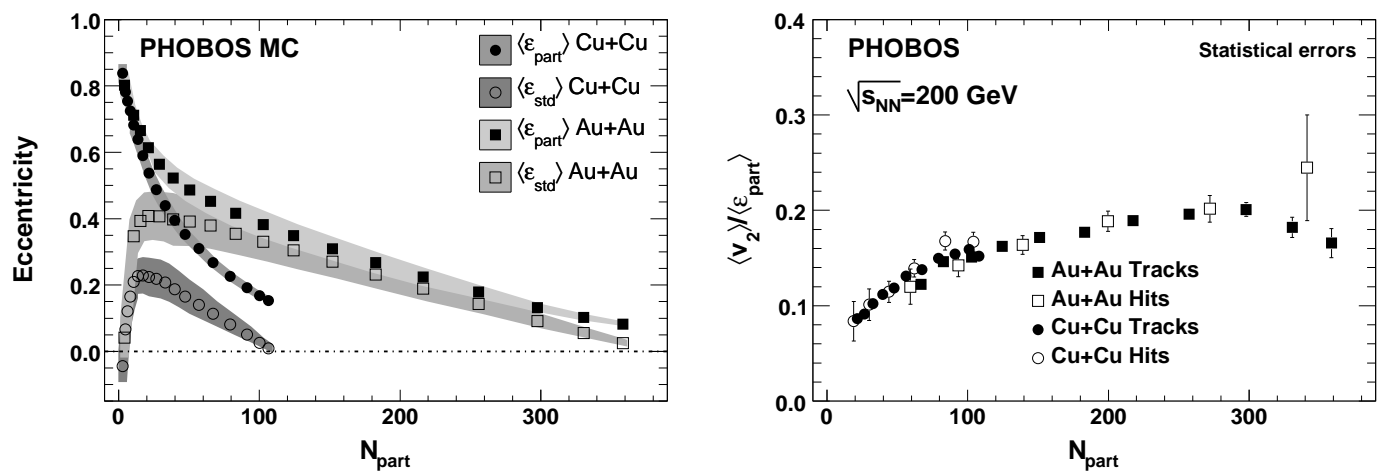

Figure 2. Left: Two different calculations for the average eccentricity for $\mathrm{Cu}+\mathrm{Cu}$ and $\mathrm{Au}+\mathrm{Au}$ collisions at $\sqrt{s_{\mathrm{NN}}}=200 \mathrm{GeV}$, see text for details. Right: The measured midrapidity $v_{2}$ divided by the participant eccentricity.

midrapidity, but also as a function of transverse momentum and pseudorapidity. This new result is shown in Figure [3, where for the same number of participants in both systems we find a consistent result out to $3.5 \mathrm{GeV} / \mathrm{c}$ in $p_{T}$ and across \pm 5 units of $\eta$.
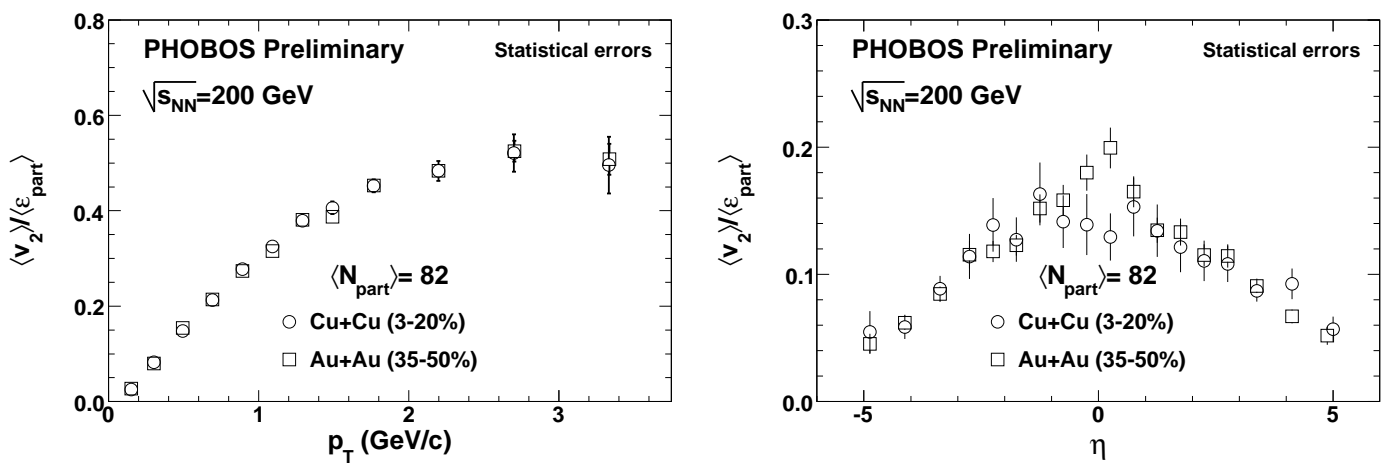

Figure 3. Eccentricity scaled elliptic flow results in $\mathrm{Cu}+\mathrm{Cu}$ and $\mathrm{Au}+\mathrm{Au}$ collisions at matched $\left\langle N_{\text {part }}\right\rangle$. Results are shown for $v_{2} /\left\langle\epsilon_{\text {part }}\right\rangle$ versus $p_{T}$ (left) and $\eta$ (right).

The apparent relevance of the participant eccentricity model in unifying the average elliptic flow results for $\mathrm{Cu}+\mathrm{Cu}$ and $\mathrm{Au}+\mathrm{Au}$ collisions leads naturally to consideration of the dynamical fluctuations of both the participant eccentricy itself as well as in the elliptic flow signal from data. Simulations of the expected dynamical fluctuations in participant eccentricity as a function of $N_{\text {part }}$ were performed using the PHOBOS Monte Carlo Glauber based participant eccentricity model, and they predict large dynamical fluctuations, $\sigma\left(\epsilon_{\text {part }}\right) /\left\langle\epsilon_{\text {part }}\right\rangle$ of the order of 0.4 in $\mathrm{Au}+\mathrm{Au}$ collisions at $\sqrt{s_{\mathrm{NN}}}=200$ $\mathrm{GeV}$. There are several different approaches one could develop to measure dynamical elliptic flow fluctuations, and PHOBOS has recently created a new method that is based on a direct measure of $v_{2}$ on an event-by-event basis using a maximum likelihood fit that utilizes the unique large pseudorapidity coverage of the PHOBOS detector [14, 18]. The strength of this approach lies in the fact that this analysis removes the effects of statistical fluctuations and multiplicity dependence by applying a detailed 

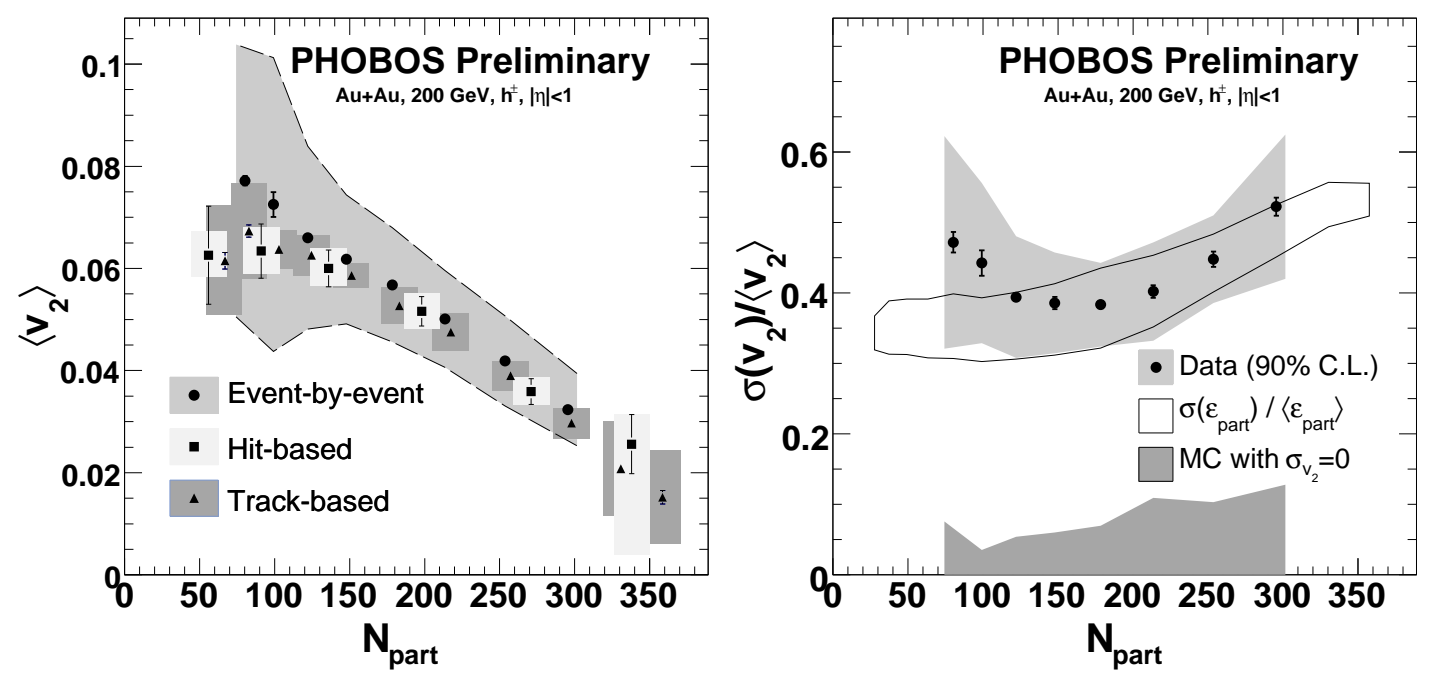

Figure 4. Left: Event-by-event measurement of $\left\langle v_{2}\right\rangle$ compared with hit and track based PHOBOS results. Right: Measured dynamical fluctuations in elliptic flow $\left(\sigma\left(v_{2}\right) /\left\langle v_{2}\right\rangle\right)$, participant eccentricity fluctuations calculated in the PHOBOS participant eccentricity model, and the sensitivity of the measurement in the case of no dynamical fluctuations.

model of the detector response that enables both a measurement of the average $v_{2}$ on an event-by-event basis as well as a measure of the dynamical fluctuations in $v_{2}$. The experimental results for both the average $\left\langle v_{2}\right\rangle$ and the measured dynamical fluctuations, which we quantify using the ratio $\sigma\left(v_{2}\right) /\left\langle v_{2}\right\rangle$, obtained in this new analysis are given in Figure 4 for $\mathrm{Au}+\mathrm{Au}$ collisions at $\sqrt{s_{\mathrm{NN}}}=200 \mathrm{GeV}$. The left-hand side of Figure 4 shows the results for the average midrapidity elliptic flow obtained from the event-by-event analysis together with the results from both the hit-based and track-based analyses. The error bars represent statistical errors and the shaded bands the $90 \%$ C.L. systematic uncertainities. Confidence that all three measurements are determining the average elliptic flow is increased through the observation that they agree within the systematic errors. The right-hand side of Figure 4 presents the new PHOBOS results for $v_{2}$ dynamical fluctuations together with the result obtained for fluctuations in the participant eccentricity. Systematics on the experimental measurement are improved by quantifying the result as a ratio of $\sigma\left(v_{2}\right) /\left\langle v_{2}\right\rangle$. We observe large dynamical fluctuations in elliptic flow with a magnitude in remarkable agreement with calculations of participant eccentricity fluctuations. This result suggests that the initial state equilibrates very rapidly with a collision eccentricity largely defined by each collision event's particular distribution of participant nucleon interaction points, and this detailed "snapshot" of the two nuclei's overlap region is propagated by the subsequent hydrodynamic evolution of the produced matter.

PHOBOS has also made great progress in studying two-particle correlations between charged particles. We have recently published results on forward-backward multiplicity correlations in non-overlapping bins of pseudorapidity for $\sqrt{s_{\mathrm{NN}}}=200 \mathrm{GeV}$ 

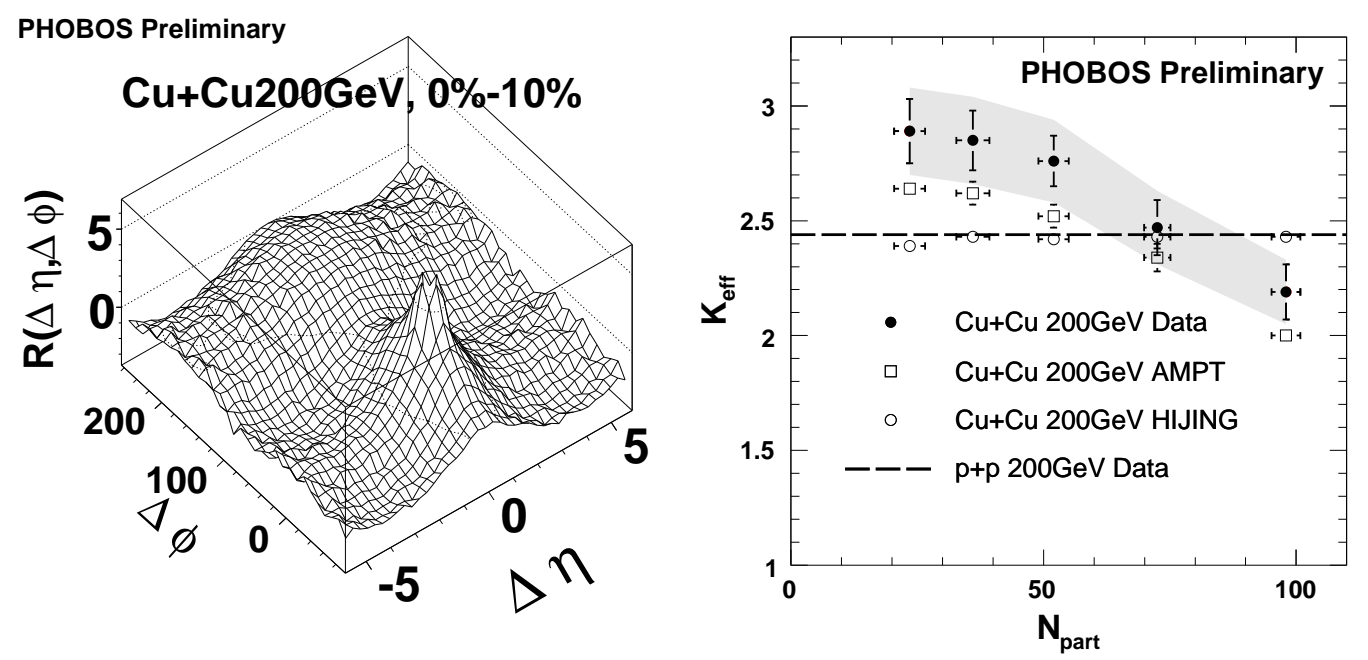

Figure 5. Left: Two particle correlation in $\Delta \eta$ and $\Delta \phi$ between all charged particles for central $\mathrm{Cu}+\mathrm{Cu}$ collisions at $\sqrt{s_{\mathrm{NN}}}=200 \mathrm{GeV}$. Right: The extracted effective cluster size, $K_{\text {eff }}$, in $\mathrm{Cu}+\mathrm{Cu}$ and $\mathrm{p}+\mathrm{p}$ data compared to HIJING and AMPT.

$\mathrm{Au}+\mathrm{Au}$ collisions [19]. From this analysis we found that particles in $\mathrm{Au}+\mathrm{Au}$ collisions are not produced independently, but instead appear to be produced in clusters. In particular, we found significant short-range correlations at all centralities, both as a function of $\eta$ and as a function of increasing pseudorapidity bin, $\Delta \eta$. Here we report a completely new two-particle correlation analysis that fully utilizes the extensive coverage in pseudorapidity $(|\eta| \leq 3.2)$ and azimuthal angle $(\Delta \phi=2 \pi)$ afforded by the PHOBOS Octagon detector and its corresponding ability to measure essentially the full bulk of charged particle emission down to very low momentum. At this point in time, we report the preliminary results of this measurement in both $\mathrm{p}+\mathrm{p}$ and $\mathrm{Cu}+\mathrm{Cu}$ collisions at $\sqrt{s_{\mathrm{NN}}}=200 \mathrm{GeV}$, where we emphasize that no $p_{T}$ cut on any particle is imposed. The correlation is calculated in the following way:

$$
R(\Delta \eta, \Delta \phi)=\left\langle(n-1)\left(\frac{F_{n}(\Delta \eta, \Delta \phi)}{B_{n}(\Delta \eta, \Delta \phi)}-1\right)\right\rangle,
$$

where $F_{n}$ is the same-event two particle correlation between all charged particles, $n$, and $B_{n}$ is the uncorrelated background. An example of the results of this analysis for central $\mathrm{Cu}+\mathrm{Cu}$ collisions is shown in the left-hand side of Figure 5. One can already observe the $\Delta \phi$ correlations resulting from elliptic flow, something not present in the corresponding $\mathrm{p}+\mathrm{p}$ data. Motivated by the interesting results found in the forwardbackward correlations analysis, we initially have focused on studying the short-range correlations in pseudorapidity. To accomplish this, we project onto $\Delta \eta$ to obtain one-dimensional correlations in $\Delta \eta$, to which we perform fits in order to extract the parameter $K_{\text {eff }}$, the effective cluster size. This result is presented in the right-hand side of Figure 5. The results clearly show that on average, even in heavy-ion collisions such as $\mathrm{Cu}+\mathrm{Cu}$, particles tend to be produced in clusters with a size of $K_{\text {eff }}=2-3$, which is surprisingly similar to that seen in elementary $\mathrm{p}+\mathrm{p}$ collisions. In addition, there is a 
nontrivial dependence of $K_{\text {eff }}$ on centrality, such that the value is larger than seen in $\mathrm{p}+\mathrm{p}$ collisions for peripheral $\mathrm{Cu}+\mathrm{Cu}$ collisions and decreases with centrality to a value below that found in $\mathrm{p}+\mathrm{p}$ for central collisions. The observed variation of $K_{\text {eff }}$ with centrality is not seen in HIJING, and although the centrality dependence is qualitatively reproduced by AMPT, the magnitude is still below that seen in the data. For more details see Ref. [15].

PHOBOS has also continued its analysis of identified charged particles. The lefthand side of Figure 6 gives one of the results for identified hadron transverse momentum spectra in $\mathrm{Au}+\mathrm{Au}$ collisions at $\sqrt{s_{\mathrm{NN}}}=62.4 \mathrm{GeV}$ [20]. The unique coverage of PHOBOS at very low $p_{T}$ is evident, and the results of a blastwave fit to the higher $p_{T}$ data, measured using both the Spectrometer and time-of-flight detector, is found to be consistent with the combined yields of pions, kaons and protons at low $p_{T}$. The right-hand side of Figure 6 shows new results on antiparticle to particle ratios as a function of centrality measured in 62.4 and $200 \mathrm{GeV} \mathrm{Cu}+\mathrm{Cu}$ [8]. The PHOBOS measurement of antiparticle to particle ratios is unique in that due to the two identical Spectrometer arms located on opposite sides of a dipole magnet we can, by simply reversing the magnetic field systematically during data taking, extract four independent measurements of identified particle ratios where all effects of acceptance and efficiency cancel out in the final ratio. The energy dependence of the proton and kaon particle ratios between $\sqrt{s_{\mathrm{NN}}}=62.4$ and $200 \mathrm{GeV}$ is clearly evident in Figure 6, and we find in all cases that the ratios are only weakly dependent, if at all, on centrality.
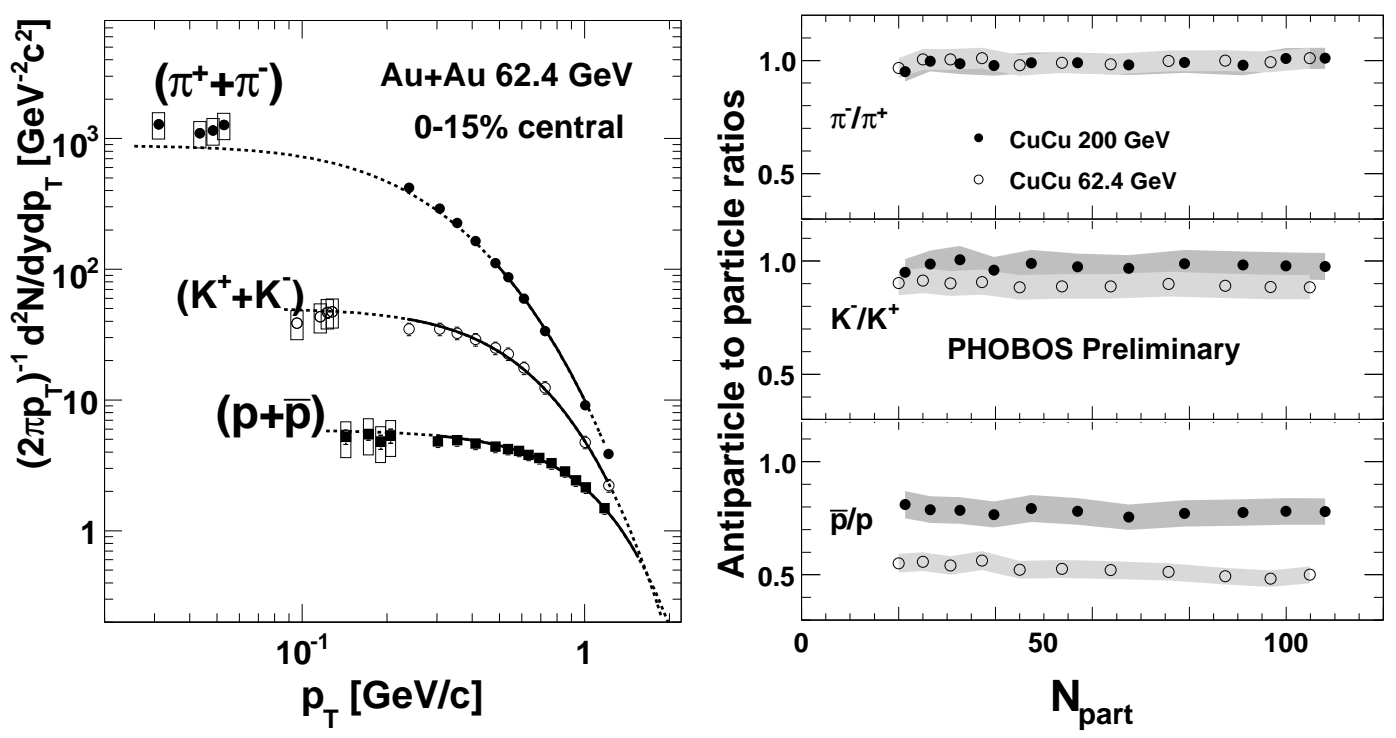

Figure 6. Left: Identified particle spectra for $\mathrm{Au}+\mathrm{Au}$ collisions at $\sqrt{s_{\mathrm{NN}}}=62.4 \mathrm{GeV}$. Right: Antiparticle to particle ratios for protons, kaons and pions in $\mathrm{Cu}+\mathrm{Cu}$ collisions at $\sqrt{s_{\mathrm{NN}}}=62.4$ and $200 \mathrm{GeV}$.

The latest results on global charged particle emission are shown in Figure 7 . As shown in the left-hand figure, we have extended the analysis for the midrapidity charged 

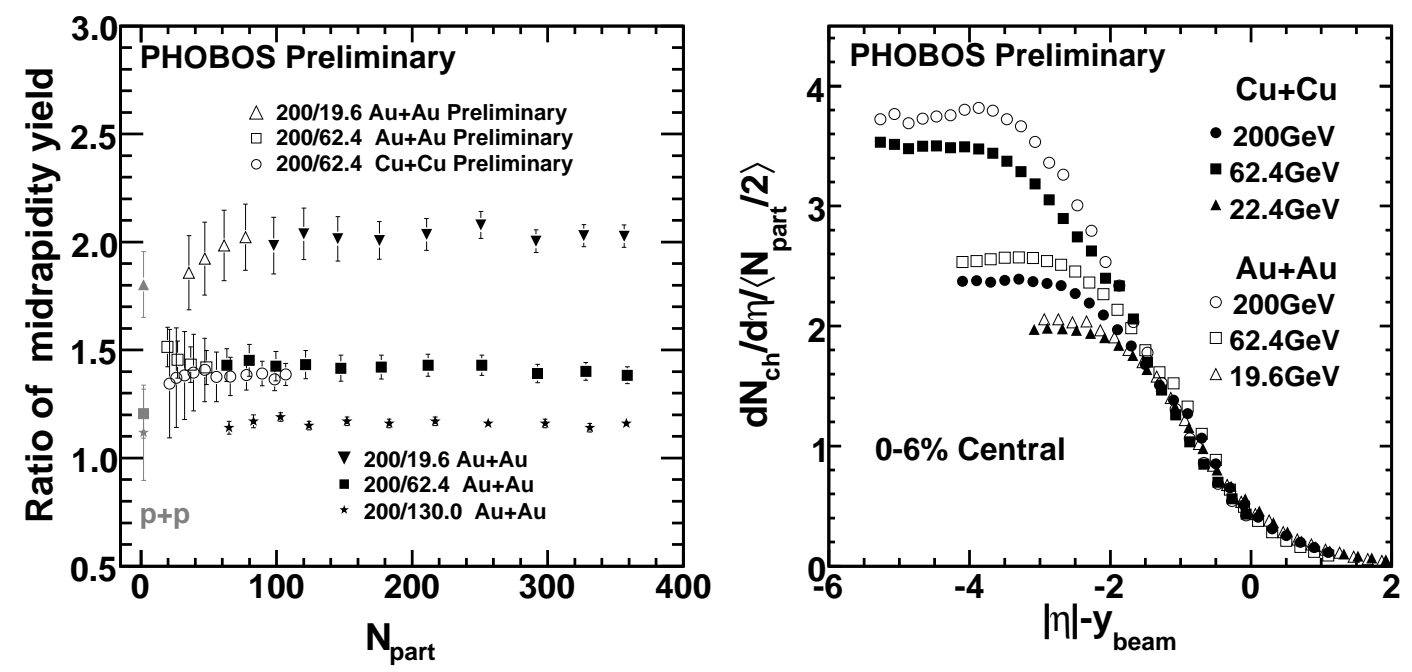

Figure 7. Global charged particle production in $\mathrm{Au}+\mathrm{Au}$ and $\mathrm{Cu}+\mathrm{Cu}$. Left: The ratio of midrapidity yields for different energies. Right: The pseudorapidity dependence for central collisions effectively shifted into the rest frame of one of the nuclei.

particle multiplicity in $\mathrm{Au}+\mathrm{Au}$ collisions to lower centrality, enabling a better overlap in $N_{\text {part }}$ with the $\mathrm{Cu}+\mathrm{Cu}$ system. On the right-hand side of Figure 7 , we have also completed the analysis of $d N_{\mathrm{ch}} / d \eta$ for all $\mathrm{Cu}+\mathrm{Cu}$ energies with the addition of the lowest energy data at $\sqrt{s_{\mathrm{NN}}}=22.4 \mathrm{GeV}$. The new results for even the lowest energy $\mathrm{Cu}+\mathrm{Cu}$ data exhibit the same striking feature of extended longitudinal scaling [5] that appears to be a general feature of all heavy-ion results at RHIC energies.

The future PHOBOS physics program will continue to be based on the comprehensive dataset that already exists. This data consists of $\mathrm{Au}+\mathrm{Au}$ collisions at $\sqrt{s_{\mathrm{NN}}}=19.6,62.4,130$ and $200 \mathrm{GeV}, \mathrm{Cu}+\mathrm{Cu}$ collisions at $\sqrt{s_{\mathrm{NN}}}=22.4,62.4$ and $200 \mathrm{GeV}, \mathrm{d}+\mathrm{Au}$ collisions at $\sqrt{s_{\mathrm{NN}}}=200 \mathrm{GeV}$ and $\mathrm{p}+\mathrm{p}$ collisions at $\sqrt{s_{\mathrm{NN}}}=200$ and $410 \mathrm{GeV}$. The $\mathrm{p}+\mathrm{p}$ dataset will allow for a measurement of $d N_{\mathrm{ch}} / d \eta$ as a function of total multiplicity. On the heavy-ion side, we will extend the studies of dynamical flow fluctuations to other systems and energies. The two-particle correlations study between all particles will also be extended to $\mathrm{Au}+\mathrm{Au}$ collisions. We are currently exploring the feasibility of performing two-particle correlations studies using a high $p_{T}$ trigger particle, as measured in the Spectrometer, correlated with the bulk charged particle production, as measured by the Octagon detector. We will continue to exploit the unique PHOBOS capabilities for particle identification at very low $p_{T}$ by using the high-statistics $\mathrm{Au}+\mathrm{Au}$ data set at $\sqrt{s_{\mathrm{NN}}}=200 \mathrm{GeV}$ to extract yields of pions, kaons and protons as a detailed function of centrality. We plan to complete the PHOBOS comprehensive study of antiparticle to particle ratios with measurements in both $\mathrm{Cu}+\mathrm{Cu}$ and $\mathrm{Au}+\mathrm{Au}$ at $\sqrt{s_{\mathrm{NN}}}=62.4$ and $200 \mathrm{GeV}$, as a detailed function of centrality and $p_{T}$. We also continue to actively pursue studies of $\phi$-meson production at very low $p_{T}$. In short, although the active data-taking of PHOBOS at RHIC has come to a close, there is still a great deal of interesting and relevant physics to be explored and discovered. 


\section{Acknowledgments}

We acknowledge the generous support of the Collider-Accelerator Department (including RHIC project personnel) and Chemistry Department at BNL. We thank Fermilab and CERN for help in silicon detector assembly. We thank the MIT School of Science and LNS for financial support.

This work was partially supported by U.S. DOE grants DE-AC02-98CH10886, DEFG02-93ER40802, DE-FG02-94ER40818, DE-FG02-94ER40865, DE-FG02-99ER41099, and DE-AC02-06CH11357, by U.S. NSF grants 9603486, 0072204, and 0245011, by Polish KBN grant 1-P03B-062-27(2004-2007), by NSC of Taiwan Contract NSC 892112-M-008-024, and by Hungarian OTKA grant (F 049823).

\section{References}

[1] Back B B et al. [PHOBOS] 2003 Nucl. Instrum. Meth. A 499603.

[2] Baker M et al. [PHOBOS] 2003 Nucl. Phys. A 71565.

[3] Back B et al. [PHOBOS] 2005 Nucl. Phys. A 75728.

[4] Roland G et al. [PHOBOS] 2006 Nucl. Phys. A 774113.

[5] Back B B et al. [PHOBOS] 2003 Phys. Rev. Lett. 91052303.

[6] Back B B et al. [PHOBOS] 2004 Phys. Rev. C 70 021902(R).

[7] Back B B et al. [PHOBOS] 2005 Phys. Rev. Lett. 94082304.

[8] Veres $\mathrm{G}$ et al. [PHOBOS], these proceedings.

[9] Back B B et al. [PHOBOS] 2002 Phys. Rev. Lett. 89222301.

[10] Back B B et al. [PHOBOS] 2006 Phys. Rev. Lett. 97012301.

[11] Nouicer $\mathrm{R}$ et al. [PHOBOS], these proceedings.

[12] Manly S et al. [PHOBOS] 2006 Nucl. Phys. A 774523.

[13] Loizides $\mathrm{C}$ et al. [PHOBOS], these proceedings.

[14] Alver B et al. [PHOBOS], Quark Matter 2006 poster, to appear in Int J Mod Phys E.

[15] Li W et al. [PHOBOS], these proceedings.

[16] Woźniak K et al. [PHOBOS], Quark Matter 2006 poster, to appear in Int J Mod Phys E.

[17] Alver B et al. [PHOBOS], Preprint nucl-ex/0610037, submitted to Phys. Rev. Lett.

[18] Alver B et al. [PHOBOS], Preprint nucl-ex/0608025, to appear in Proceedings of Science.

[19] Back B B et al. [PHOBOS] 2006 Phys. Rev. C 74 011901(R).

[20] Back B B et al. [PHOBOS], Preprint nucl-ex/0610001, accepted for publication in Phys. Rev. C. 